Universidad de Lima

Facultad de Ciencias Empresariales y Económicas

Carrera de Economía

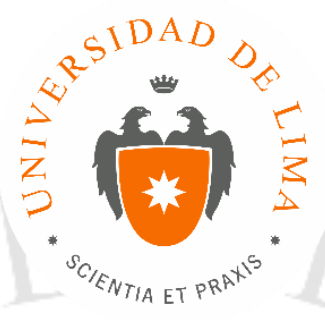

\title{
APLICACIÓN DEL MODELO DE \\ GRAVEDAD PARA EL ANÁLISIS DE LOS \\ DETERMINANTES DE LAS \\ EXPORTACIONES PERUANAS HACIA LA UNIÓN EUROPEA 2000-2017
}

Trabajo de suficiencia profesional para optar el Título Profesional de Economista

Sustentación de caso

Israel Laos Euscategui

Código 20130682

Asesor

José Manuel Azañero Saona

Lima - Perú

Diciembre de 2018 


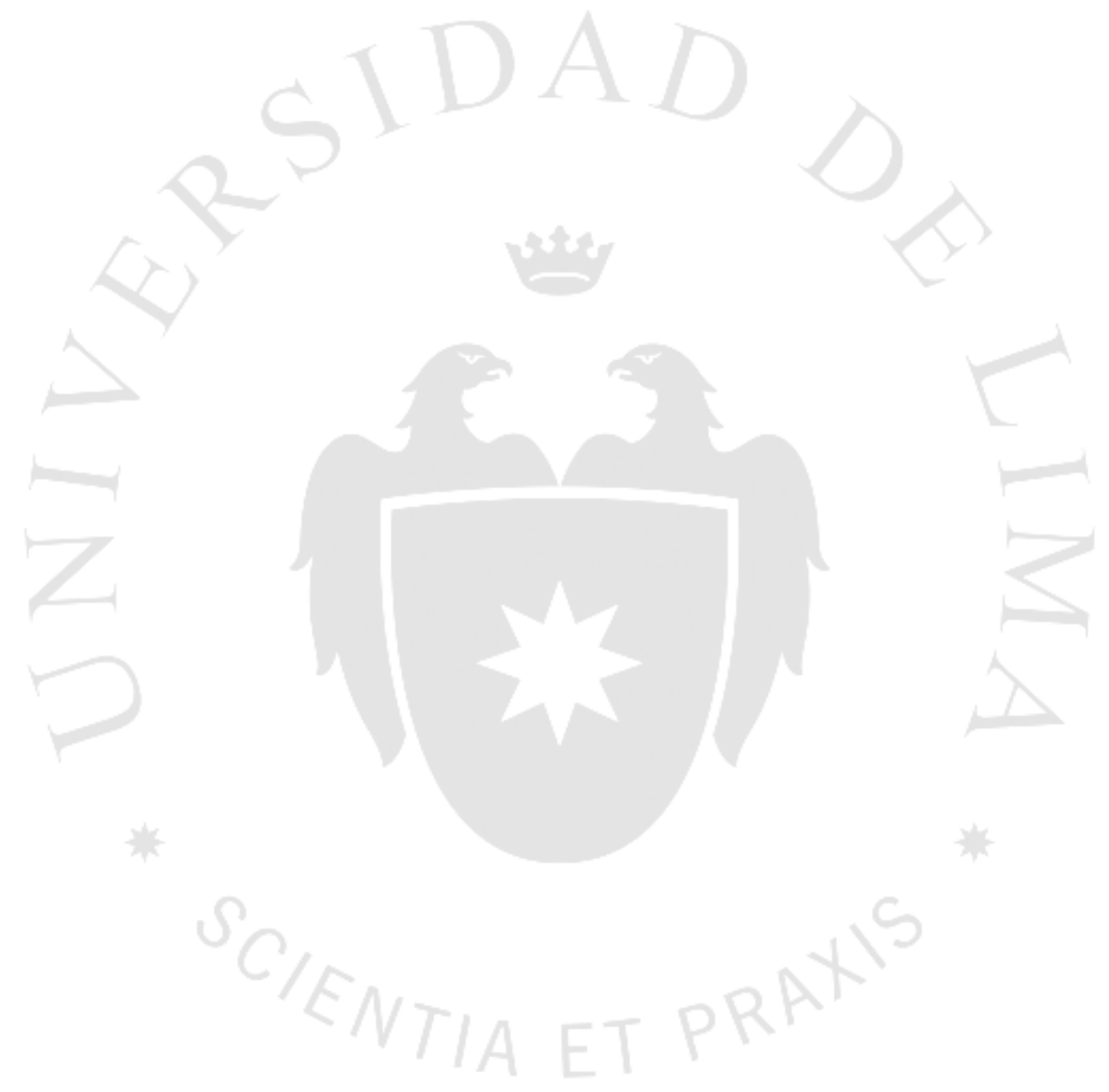




\section{APLICACIÓN DEL MODELO DE GRAVEDAD PARA EL ANÁLISIS DE LOS DETERMINANTES DE LAS EXPORTACIONES PERUANAS HACIA LA UNIÓN EUROPEA 2000-2017}




\section{TABLA DE CONTENIDO}

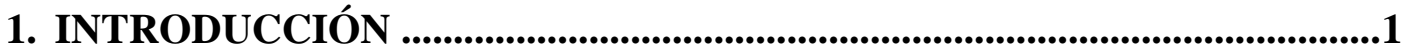

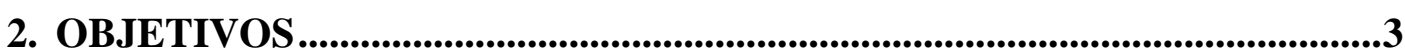

3. PREGUNTAS DE INVESTIGACIÓN................................................................4

4. METODOLOGÍA DE RECOLECCIÓN DE LA INFORMACIÓN ...............5

5. METODOLOGÍA DE ANÁLISIS DE LA INFORMACIÓN...........................7

6. DESCRIPCIÓN DE CASO ..............................................................................12

6.1 Exportaciones peruanas hacia la UE.................................. 12

6.2 Producto Bruto Interno ........................................................ 13

6.3 Distancia...................................................................... 14

6.4 Landlocked ................................................................. 15

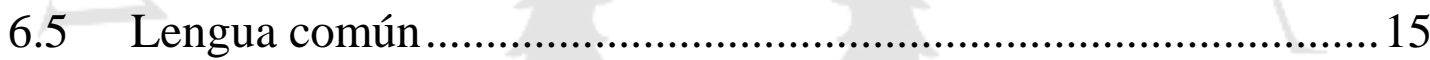

6.6 Tipo de cambio real bilateral .............................................. 16

6.7 Acuerdo comercial ............................................................. 16

6.8 Términos multilaterales de resistencia comercial .......................16

7. ANÁLISIS Y DISCUSIÓN DE RESULTADOS ...............................................17

RECOMENDACIONES ................................................................................ 21

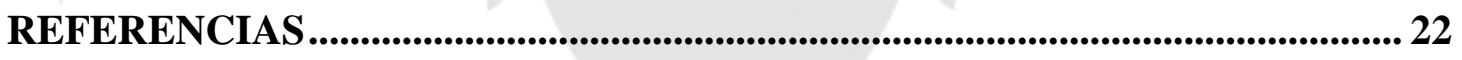

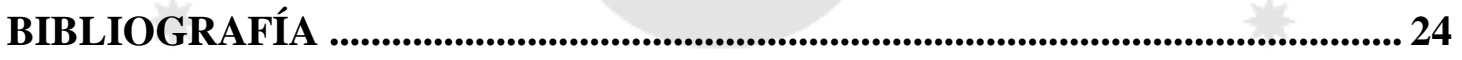




\section{ÍNDICE DE TABLAS}

Tabla 7.1 Resultados econométricos

19 


\section{ÍNDICE DE FIGURAS}

Figura 1.1 Relación comercial entre Perú y UE (28).................................................... 1

Figura 1.2 Índice de Herfindahl y Hirschman y productos exportados .........................2

Figura 6.1 Composición de las exportaciones ........................................................... 13

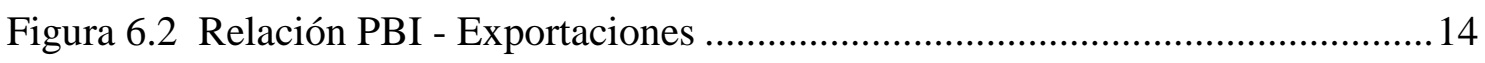

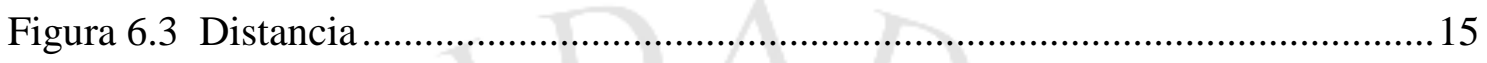




\section{ÍNDICE DE ANEXOS}

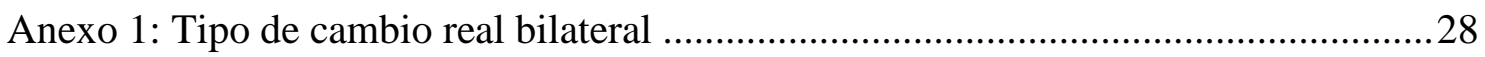

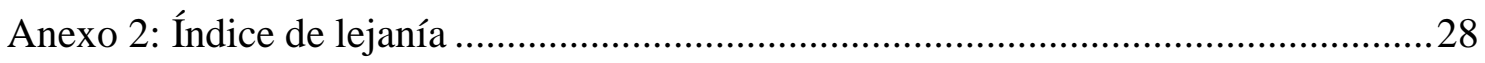

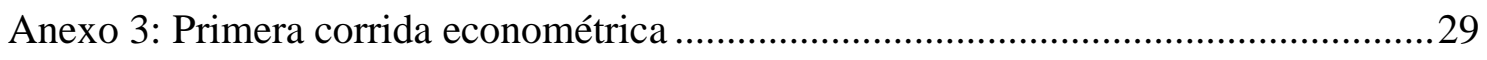

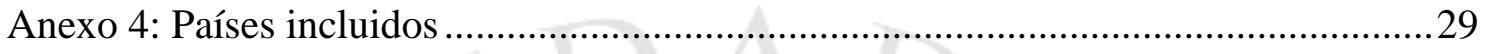

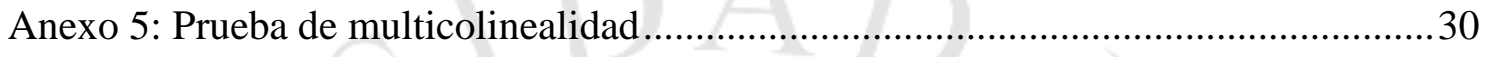

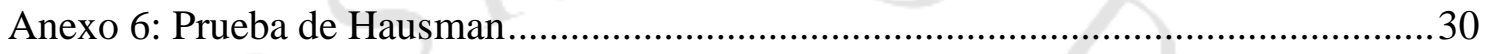

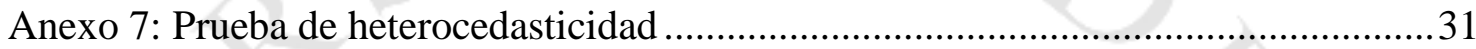

Anexo 8: Prueba de autocorrelación de primer orden ................................................ 31

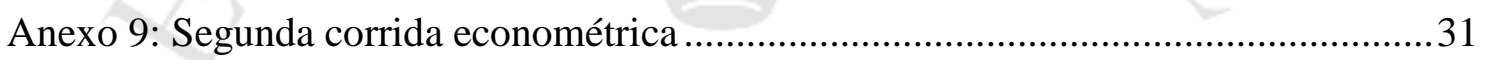




\section{INTRODUCCIÓN}

La relación comercial entre el Perú y los veinte y ocho que conforman la Unión Europea (UE) se ha ido fortaleciendo entre los años 2000 y 2017. Una muestra de ello es el acuerdo comercial que entró en vigencia el primero de marzo del 2013 y el incremento de las exportaciones e importaciones peruanas a lo largo del periodo de estudio. Como se observa en el Figura 1.1, las exportaciones peruanas en los años 2000 y 2017 fueron de \$ 1,050 y \$ 6,531 millones respectivamente, así mismo, en el año 2011 se registró la exportación más alta del periodo de estudio, \$ 8,702 millones; por otro lado, las importaciones peruanas en los años 2000 y 2017 fueron de \$1,040 y \$4,869 millones respectivamente, siendo el año 2013 con las importaciones más altas de periodo, $\$ 5,200$ millones. Este escenario ha sido favorable para el Perú ya que su balanza comercial siempre ha estado positiva, es decir, sus exportaciones hacia la Unión Europea han sido mayores que sus importaciones; es así que esta brecha se ha mantenido por encima de $\$$ 1,000 millones a partir del año 2002 hasta el 2017, siendo el año 2011 con mayor diferencia, \$ 4,591 millones. De esta manera, la Unión Europea llega a representar, en promedio, cerca del $19 \%$ del total de las exportaciones peruanas, lo que le asigna como uno de los principales mercados de destino de exportación.

\section{Figura 1.1}

Relación comercial entre Perú y UE (28)

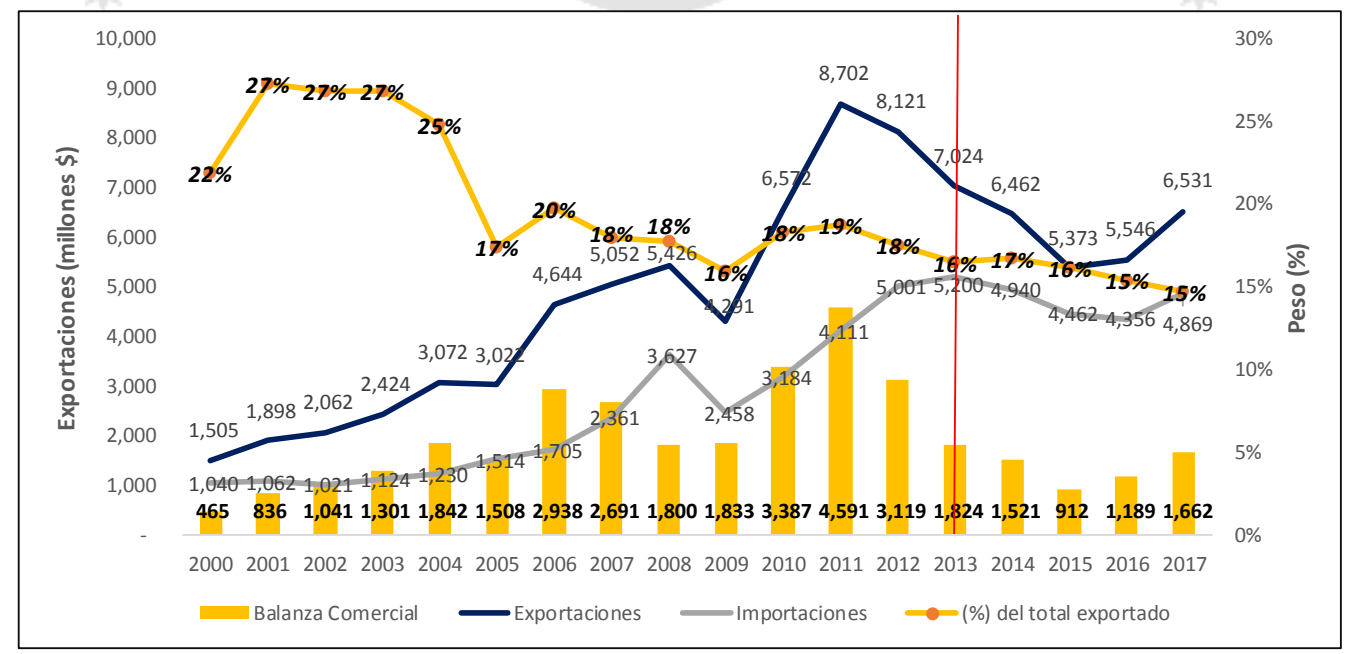

Nota: Acuerdo comercial entre el Perú y la Unión Europea en el 2013 Fuente: Elaboración propia / Adex Data Trade 
Por otro lado, los productos más exportados a la UE a lo largo de periodo de estudio están los minerales de cobre y sus concentrados (19.2\%), cátodos y secciones cátodos de cobre refinados $(8.7 \%)$, oro $(8.3 \%)$, minerales de cinc y sus concentrados $(6.0 \%)$ y demas de café sin descafeinar, sin tostar (5.9\%). Sin embargo, a pesar que el $50 \%$ de lo exportado de centra en 5 productos, no se observa una concentración alta; por ejemplo, en la Figura 1.2, el índice de Herfindahl y Hirschman, que indica el grado de concentración dentro de un rango entre 0 y 10,000, siendo cero una señal que no existe concentración y diez mil lo contrario, afirma que en entre los años 2000 y 2003 hubo concentración moderada en las exportaciones peruanas, y en los años posteriores no hubo concentración. Además, el número de empresas que exportan más de \$1,000 a la Unión Europea crecieron en 78\% desde el año 2000 al 2017, entre ellas, se encuentran Southern Perú Cooper Corporation, Compañía minera Antamina, Minsur, Perú LNG y Sociedad Minera Cerro Verde.

Figura 1.2

Índice de Herfindahl y Hirschman y productos exportados

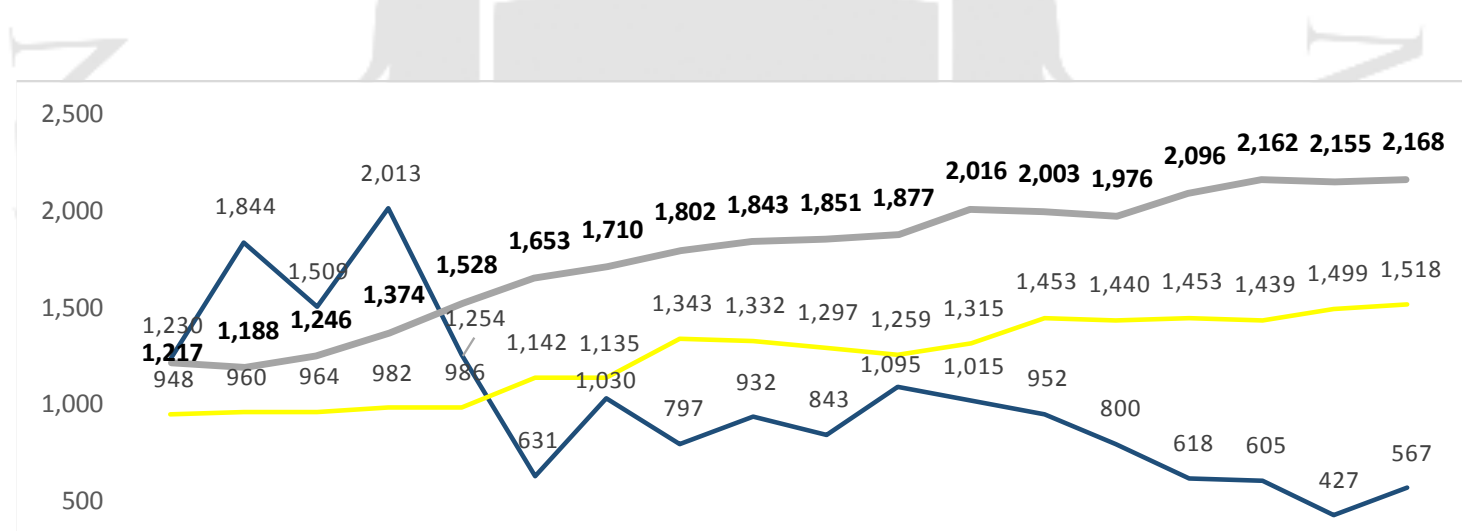

200020012002200320042005200620072008200920102011201220132014201520162017 $\longrightarrow \mathrm{HHI} \longrightarrow$ Productos $(*) \longrightarrow$ Empresas $(* *)$

Nota: (*) Se consideró partidas arancelarias de 10 dígitos con exportaciones mayores a $\$ 1,000$. (**) Se consideró las empresas que exportaron más de \$1,000 anualmente Fuente: Elaboración propia / Adex Data Trade 


\section{OBJETIVOS}

Para empezar, se presentará el objetivo general y los objetivos específicos de esta investigación.

\section{Objetivo General}

- El objetivo general de la presente investigación es comprender los factores determinantes de las exportaciones peruanas, en el periodo comprendido desde el año 2000 al 2017 hacia los veinte y ocho países que pertenecen a la Unión Europea.

Objetivos Específicos

- Determinar si el acuerdo comercial entre el Perú y la Unión Europea, que entró en vigencia a partir del primero de marzo del año 2013, ha sido significativo y ha tenido efectos positivos en las exportaciones peruanas a dicho destino.

- Entender si el tipo de cambio real bilateral entre el Perú y los países que incorporan la Unión Europea ha sido significativo y ha tenido efectos negativos sobre las exportaciones peruanas a los países mencionados. 


\section{PREGUNTAS DE INVESTIGACIÓN}

A continuación, se presentará la pregunta general y las preguntas específicas de la investigación.

\section{Pregunta General}

¿Cuáles son los principales factores determinantes significativos de las exportaciones peruanas hacia los países que conforman la Unión Europea en el periodo comprendido entre el año 2000 y 2017 ?

\section{Preguntas Específicas}

¿Es posible que el acuerdo comercial entre el Perú y la Unión Europea, vigente a partir del primero de marzo del año 2013, ha sido significativo y ha tenido un efecto positivo sobre las exportaciones peruanas a partir de la fecha hasta el año 2017 ?

¿Es posible que el tipo de cambio real bilateral entre el Perú y los países que forman parte de la Unión Europea ha sido significativo y ha tenido efectos negativos sobre las exportaciones peruanas en el periodo de estudio? 


\section{METODOLOGÍA DE RECOLECCIÓN DE LA INFORMACIÓN}

En el siguiente apartado se desarrollará el proceso de análisis de las variables incluidas en el modelo econométrico. Cabe recalcar que las variables usadas en la presente investigación son información secundaria puesto que se obtuvo a través de distintas fuentes de acceso abiertos para países como España, Países Bajos, Alemania, Reino Unido, Italia, Bélgica, Bulgaria, Francia, Dinamarca, Suecia, Suecia, Polonia, Portugal, Finlandia, Irlanda, Grecia, Eslovaquia, Lituania, Austria, Rumania, Estonia, Hungría, República Checa, Eslovenia, Croacia, Malta, Chipre, Letonia y Luxemburgo.

En primer lugar, las exportaciones peruanas con destino a los veinte y ocho países pertenecientes a la UE se extrajeron de la fuente Adex Data Trade (ADT). Se usó la información desde el año 2000 al 2017 considerando todos los sectores económicos. Los datos están en dólares y a precios actuales.

En segundo lugar, el Producto Bruto Interno (PBI) del Perú y de los países de la UE se obtuvo de la base de datos del Banco Mundial (BM) y se utilizó la información desde el año 2000 al 2017. Los datos se encuentran en dólares y a precios actuales.

En tercer lugar, el PBI per cápita del Perú y los países de la UE se obtuvieron de la base de datos del BM desde el año 2000 al 2017.

En cuarto lugar, la distancia entre el Perú y los socios comerciales de la UE se obtuvieron de la fuente de datos del Centre d'Etudes Prospectives et d'Informations Internationales (CEPII). La distancia está expresada en kilómetros y se considera la lejanía entre los puntos céntricos de cada capital de los países analizados.

En quinto lugar, las relaciones comerciales entre el Perú y la UE es una variable dicotómica que tiene el valor de uno a partir del 2013 al 2017, y cero en los años restantes. La información se obtuvo de la página Acuerdos Comerciales del Perú del Ministerio de Comercio Exterior y Turismo (MINCETUR).

En sexto lugar, la variable Landlocked o país sin acceso a la costa es una variable dummy que toma el valor de uno si el socio comercial no tiene acceso al mar y cero en caso contrario. La información se obtuvo del CEPII. 
En séptimo lugar, la variable Common Language o lengua común es una variable dicotómica que toma la unidad si el Perú y el país que pertenece a la Unión Europea comparten la misma lengua, y cero en caso contrario. La información se obtuvo del CEPII.

En octavo lugar, la variable Tipo de Cambio Real Bilateral (TCRB) se obtuvo de diferentes fuentes ya que es un indicador construido siguiendo la fórmula de la Guía Metodológica de la Nota Semanal del Banco Central de Reserva del Perú (Anexo 1). El tipo de cambio nominal del Sol respecto a la unidad monetaria del país de la Unión Europea se obtuvo de la base de datos de la United Nations Conference on Trade and Development (UNCTADSTAT) y los índices de precios del consumidor del World Economic Outlook (WEO) para los años correspondientes de cada país.

Finalmente, las variables llamadas términos multilaterales de resistencia al comercio (TMRC) se construyeron siguiendo el concepto de lejanía otorgado por Head y Mayer en el 2003 (Anexo 2) con tres variables, la distancia entre el país exportador e importador, el PBI del país importador y el PBI del mundo para cada año correspondiente. La primera se consiguió de la fuente de datos del CEPII y las restantes del BM. 


\section{METODOLOGÍA DE ANÁLISIS DE LA INFORMACIÓN}

En esta sección se procederá a analizar la metodología usada para obtener los datos y los resultados de la ecuación propuesta, la teoría económica y las herramientas cualitativas y cuantitativas utilizadas.

Primero se procederá a indicar cómo se estructuró la base de datos usada. La información fue administrada a través de una base de datos construida a partir de fuentes de información estadísticas provenientes de instituciones reconocidas como La Asociación de Exportadores (ADEX), Banco Central de Reserva, Banco Mundial, CEPII, Fondo Monetario Internacional (FMI), MINCETUR y UNCTADSTAT, y será depositada en un documento en Excel con el fin de exportarlas al programa Stata14 para proceder con las estimaciones econométricas.

Segundo, se desarrollará la base teórica del modelo de gravedad utilizada en la presente investigación. En un inicio, según (Correia, 2008), la ecuación de gravedad fue propuesta en 1687 por Isaac Newton a través de la ley gravitacional universal la cual explica la atracción existente entre dos masas (p. 174). Esta está formulada de la así:

$$
\mathrm{Fij}_{\mathrm{ij}}=\mathrm{G}^{*} \underline{\mathrm{MiM}_{\mathrm{i}}}
$$

$\mathrm{Dij}_{\mathrm{ij}}$

Fij: Fuerza de atracción entre i y j

G: Constante gravitacional

Mi Mj: Masas de i y j

Dij: Distancia entre i y j

Después de ello, según (Bacaria-Colom, Osorio-Caballero, \& Artal-Tur, 2013) las primeras investigaciones que realizaron modelos econométricos relacionados a los flujos comerciales entre dos países, aplicando la ley de la gravitación universal, fueron realizados por Tinbergen en el año 1962 (p. 151); así mismo, Pöyhönen (1963) fue otro 
pionero en utilizar el modelo de gravedad. De esta manera, la forma básica del modelo de gravedad del comercio desarrollado Tinbergen es el siguiente:

$$
F_{i j}=G * \underline{Y_{i} Y_{j}}
$$

$\mathrm{D}^{2_{\mathrm{ij}}}$

Fij: Flujo bilateral entre el país i y j.

G: Constante gravitacional.

YiYj: PBI del país i y j.

Dij: Distancia entre los países i y j.

Este modelo explica los determinantes de los flujos del comercio, implica la relación directa entre el PBI de los países que comercian y es inversa respecto a la distancia entre ellos. Años después, Linnemann (1996) incluye, a su ecuación, la variable población al modelo de gravedad con el motivo de revelar el papel de las economías de escala; por lo tanto, la ecuación de gravedad es la siguiente:

$$
X_{i j t}=B_{0} Y_{i t} Y_{j t} N_{i t} N_{j t} D_{i j}
$$

La variable $X_{i j t}$ representa el flujo de comercio entre los países i y j en el año t, Yit e Yjt es el PBI de los países i y j en el tiempo t, Nit y Njt es la población del país i y j en el tiempo t, y Dij es la distancia entre el país i y j. Respecto a la ecuación anterior, se han otorgado diferentes interpretaciones que han dado ambigüedad a los signos esperados; por ejemplo, (Sierra Fernández \& Martínez Campillo, 2009) indican que, primero, a mayor población, más diversificada su economía y, por ende, menor compromiso comercial; segundo, es posible obtener una economía de escala y especialización productiva, por lo tanto, el país estará más activamente en el comercio internacional C 10). 
A pesar de ello, se ha tratado de encontrar justificación teórica a la ecuación de gravedad en relación a los diferentes temas relacionados al comercio internacional. El primero en abordar el tema fue Anderson (1979) seguido por Bergstrand $(1985,1989)$ y Helpman (1987); el primero de ellos explicó que la ecuación mencionada se puede deducir usando el supuesto de Armington (1969) el cual indica las propiedades de los modelos del gasto a través de bienes diferenciados, y en el que todos los estados tienen una función de utilidad igual; el segundo, partiendo del supuesto de la diferenciación de las empresas por país de origen, indica que la ecuación de gravedad es una derivación de un subsistema de equilibrio parcial procedente de un modelo de equilibrio general del comercio mundial, es decir, es un híbrido entre el modelo de Heckscher-Ohlin, que supone competencia perfecta y homogeneidad de bienes, y el modelo de Competencia Monopolística. Así mismo, (Deardorff, 1995) señala que el éxito se debe a que cualquier modelo de comercio internacional elaborará una ecuación parecida (p. 10).

Por otro lado, hubo otros intentos para obtener justificación teórica de la ecuación de gravedad, uno de ellos es la demostración, en el trabajo Krugman (1995) y Helpman y Krugman (1985), que la ecuación es, posiblemente, producto del modelo de competencia monopolística con rendimientos crecientes a escala. Deardoff (1998) demuestra que la ecuación de gravedad probablemente sea armonizable en el modelo Ricardiano y en el de Heckscher-Ohlin. De igual manera, Evenet y Keller (2002) mencionaron que la ecuación de gravedad, bajo los supuestos de especialización perfecta e imperfecta del producto, fue resultante de la teoría de Heckscher-Ohlin y de la hipótesis de rendimientos crecientes a escala. Por otro lado, Eaton y Kortum (2002) transformó un modelo Ricardiano de comercio de bienes homogéneos que se ajusta a la ecuación.

Posteriormente, a lo largo de las siguientes investigaciones sobre la ecuación de gravedad, los autores han ido agregando variables al modelo, relacionadas al comercio exterior, en búsqueda de mejores resultados. Como se indica en el trabajo de (Head, 2000) la mayoría de autores añaden otras variables con menos justificación teórica pero que, basado en la experiencia, han mostrado que son importantes en el modelo (p. 8). Las variables expresadas en el trabajo de Head (2000) son PBI per cápita, Continuidad, Relaciones coloniales o en idioma y Frontera; así mismo, incluyen variables como acuerdos comerciales y monetarios. Un reflejo de ello son los trabajos de Correia (2008), Sierra y Martínez (2009), Bacaria-Colom et al. (2013), Serrano, Martínez, Rodríguez y 
Salazar (2015), Ordoñez, K. Rojas, C. y Rojas, Y (2015) y Urcia (2016) quienes han incluido las variables mencionadas y han encontrado relevancia en ellas.

Por otro lado, algunos autores han utilizado variables temporales en sus estimaciones. Sin embargo, en el presente trabajo no se incluirán ya que es un enfoque de único país. Respecto al enfoque multi-país y país-único (Földvár, 2006) indica que en el enfoque de país único se suelen evaluar dos ecuaciones ,tanto para exportaciones e importaciones; así mismo, es necesario omitir variables dummys temporales si se desea una adecuada interpretación de las variables explicativas que varían a través del tiempo (p. 54).

Tercero, el tratamiento econométrico del caso de estudio es de datos de panel puesto que la investigación es de corte transversal y longitudinal.

Un modelo de datos de panel incluye una muestra de agentes económicos para un periodo determinado, combinando los datos de tipo temporal y estructural. El principal objetivo de aplicar los datos de panel es para detectar la heterogeneidad no observable ya sea en los agentes económicos o en el tiempo, también permite un análisis más dinámico incorporando el tiempo en los datos. Para (Mayorga \& Muñoz, 2000) se analizan principalmente dos aspectos que forman parte de la heterogeneidad no observable: los efectos individuales específicos, los cuales no influyen de manera similar a cada agente y los efectos temporales, cuyo efecto es igual (p. 3).

De igual modo, (Mayorga \& Muñoz, 2000) mencionan que los datos de panel permiten observaciones más altas, incrementa los grados de libertad y reduce la colinealidad de las variables, captura la heterogeneidad no observable e incorpora el estudio series de tiempo juntamente con el de corte transversal (p. 5).

A continuación, se presenta la formulación genérica para los datos de panel.

$$
y_{i t}=\alpha+\beta^{\prime} X_{i t}+\mu_{i t}+e_{i t}
$$

En primer lugar, para verificar si las variables tienen problema de estacionariedad se realizarán las pruebas de raíz unitaria de Levin-Lin-Chu, Harris-Tzavalis, Im-PresaranShin, Fisher, Dickey fuller y Phillips-Perron. Así mismo, para identificar el problema de 
multicolinealidad, es decir, la correlación entre las variables independientes, se usará la prueba VIF. Por otro lado, para identificar si el modelo es un panel de datos de efectos aleatorios o de efectos fijos, se usará la prueba de Hausman para identificar, estadísticamente, qué tipo de panel data seguir. Luego, se realizará la prueba de heterocedasticidad para analizar si la varianza de las perturbaciones no es constante a lo largo de las observaciones, si el test indica que existe el problema de heterocedasticidad se procederá a corregirlo para que los estimadores sean robustos. Después, se realizará la prueba de autocorrelación serial para identificar si los términos de error del modelo no son independientes entre sí; en caso que se encuentre problema de autocorrelación se realizará la correlación para obtener estimadores eficientes. Así mismo, se utilizará la prueba de p-valor, esta se usará para determinar si una variable es significativa, la hipótesis nula indica que la variable no es significativa para el modelo; también se usará la prueba F para observar si las variables, en conjunto, son significativas. Por último, se realizará la prueba del R cuadrado ajustado para identificar la explicación de las variables dependientes sobre la independiente. 


\section{DESCRIPCIÓN DE CASO}

La presente investigación busca analizar la importancia que tienen los determinantes de las exportaciones peruanas hacia la Unión Europea en el periodo comprendido entre los años 2000 - 2017 bajo el modelo de gravedad.

La relación comercial entre el Perú y la zona económica ha tomado mayor importancia para el país latinoamericano ya que la UE es una de las economías con mayor bonanza; esta representa la tercera en comercio y quinta en producción mundial. Tiene un PBI per cápita de US\$ 41,2 mil, destacando los ingresos de Luxemburgo, Irlanda y Países Bajos (MINCETUR, 2018, p.1).

Así mismo, la relación comercial entre el Perú y la UE tuvo como origen la negociación previa entre la Unión Europea y la Comunidad Andina (CAN); sin embargo, dicha negociación fue suspendida en el 2008. Cinco años después, junto a Colombia, en el año 2013, entró en vigencia el acuerdo comercial Perú - Unión Europea. El (MINCETUR, 2018a) señaló que el acuerdo es parte de una estrategia a favor de Perú para convertirlo en un país exportador, consolide mercados y forme una oferta exportable competitiva (párr. 5).

Sin embargo, hubo, además el acuerdo comercial, otros factores que influyeron en el incremento de las exportaciones peruanas hacia la UE en el periodo de estudio. Por lo tanto, es necesario describir las posibles variables que fueron significativas para el objetivo general.

\subsection{Exportaciones peruanas hacia la UE}

Las exportaciones del Perú hacia la Unión Europea representaron, en el 2011, el 19\% del total exportado y es el tercer mercado más importante después de China y Estados Unidos. Así mismo, Alemania, Inglaterra, Francia e Italia son los principales destinos, dentro de las exportaciones peruanas. Por otro lado, en la Figura 6.1 se observa que la estructura de las exportaciones en el año 2000 estaba compuesto por sectores tradicionales $(74 \%)$ y no tradicionales (26\%), estas estaban compuestas principalmente por la minería tradicional $(72 \%)$ y pesca tradicional (17.1\%), y agropecuario e industrias 
(39.8\%) y pesca (18.8\%) respectivamente. Sin embargo, en el año 2017 la estructura cambió ya que el sector no tradicional tuvo una mayor participación (42\%) frente al sector tradicional (58\%); la primera está compuesta por la minería tradicional (69\%) y petróleo y gas natural (16.5\%), la segunda, por agropecuario e industrias $(65 \%)$ y pesca $(11.9 \%)$.

Figura 6.1

Composición de las exportaciones

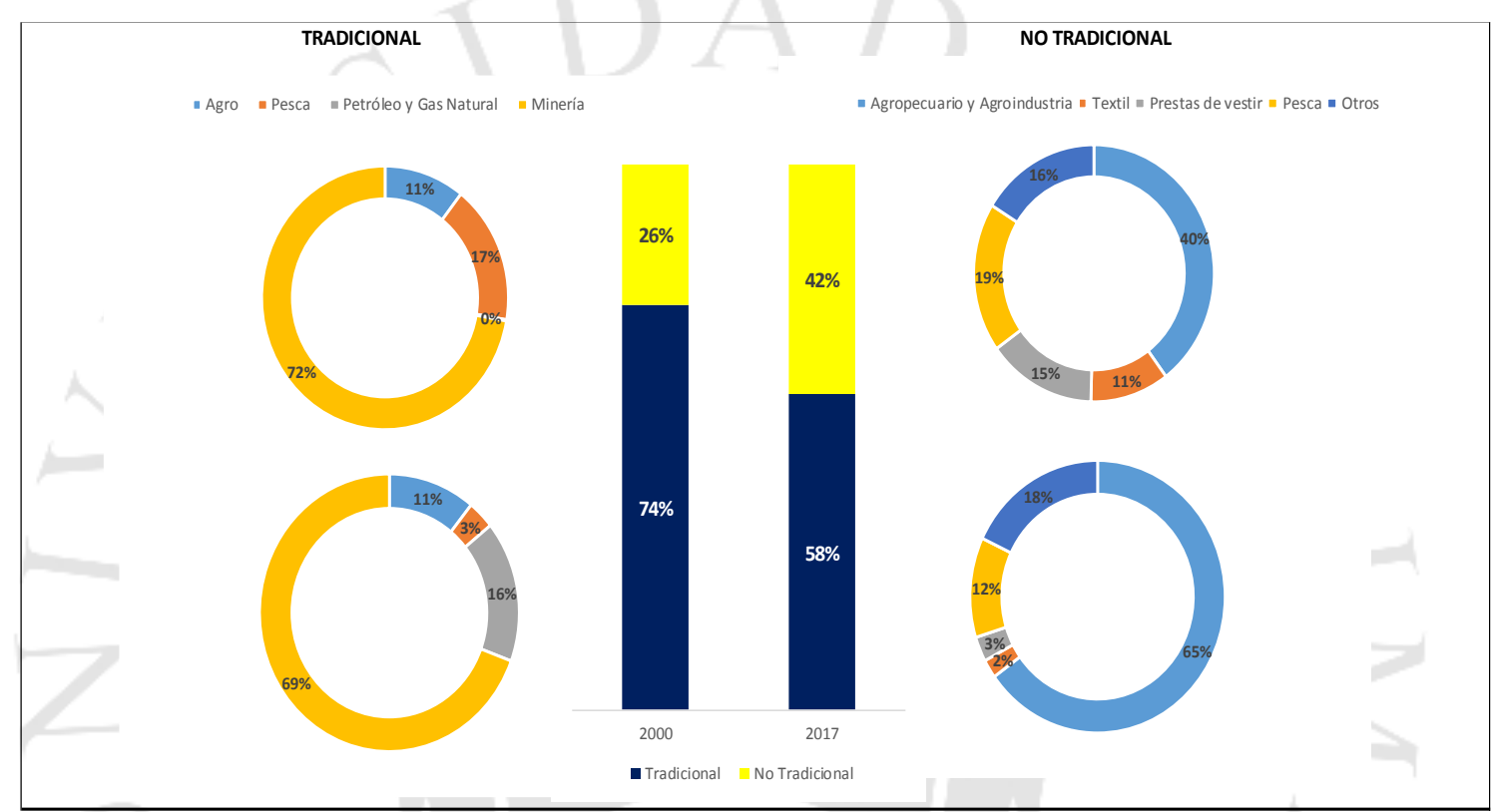

Fuente: Elaboración propia / Adex Data Trade

\subsection{Producto Bruto Interno}

El PBI es utilizado frecuentemente en los modelos de gravedad ya que representa el tamaño de una economía. Además, (Krugman, Obstfeld, \& Melitz, 2012) indican que existen relaciones positivas entre el tamaño de un país sobre el comercio internacional (p. 12). La ejemplificación de lo mencionado se puede observar en la Figura 6.2 debido a que, en el 2017, las exportaciones peruanas se concentraron en España, Alemania, Inglaterra, Italia, Bélgica y Holanda, y dichos países son los que tienen mayor producto bruto interno en la Unión Europea. 
Figura 6.2

Relación PBI - Exportaciones

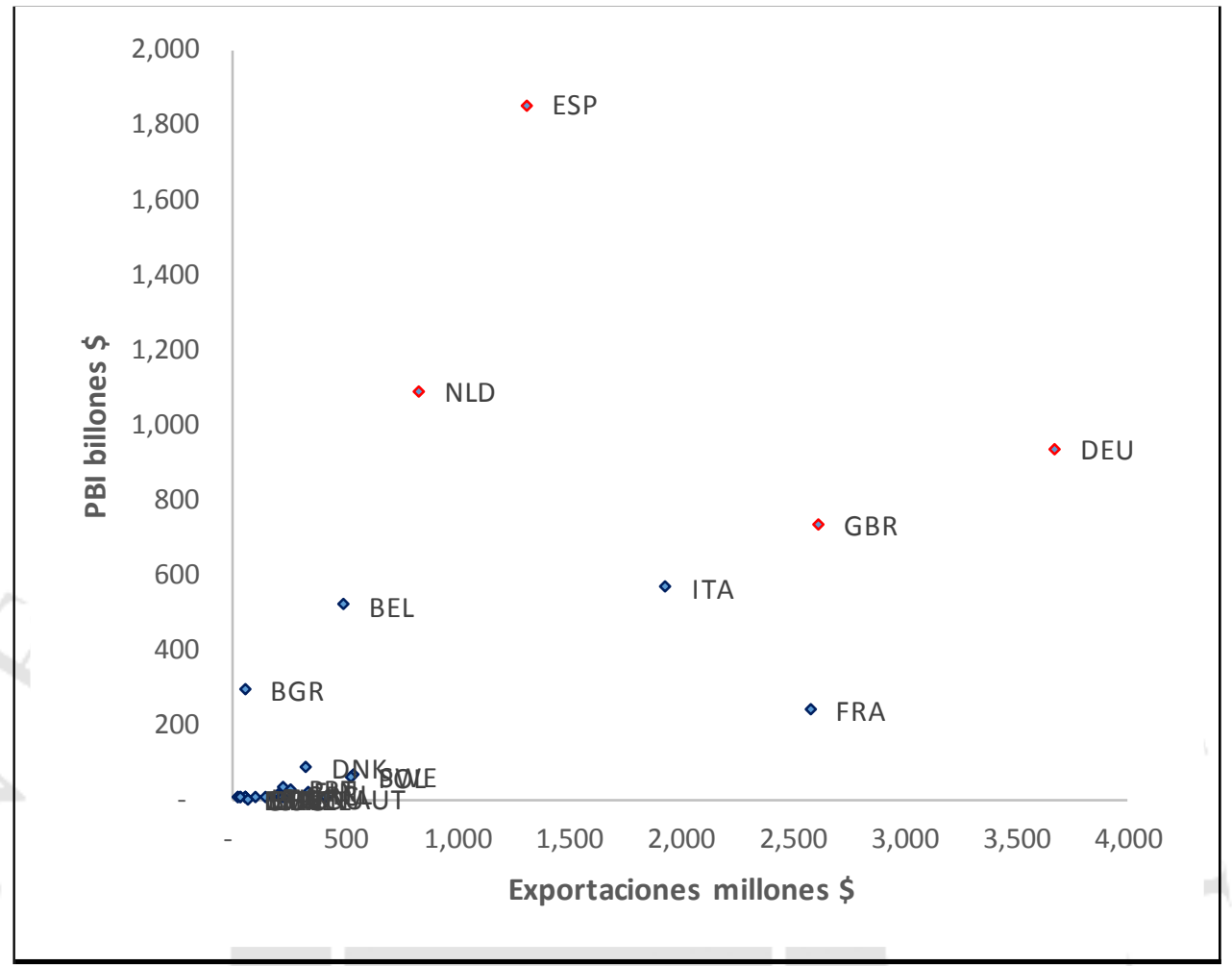

Fuente: Elaboración propia / Adex Data Trade

\subsection{Distancia}

La distancia es una aproximación de los costos de transacción, es decir, será más costoso llevar el bien o servicio de un país hacia otro si la distancia entre ambos es mayor. (Head, 2000) señala que la distancia usualmente es medida usando la metodología del gran círculo. Esta fórmula asume la forma de la Tierra como una esfera y calcula la distancia mínima a lo largo de la superficie (p. 5). En la Figura 6.3 se puede observar la relación inversa entre distancia y exportaciones; por ejemplo, la distancia entre el Perú y Chipre es la más alta entre los países de la UE, y las exportaciones peruanas hacia dicho destinos son las más bajas. Por otro lado, España fue el principal importador de productos peruanos y es el país con menor distancia. 
Figura 6.3

Distancia

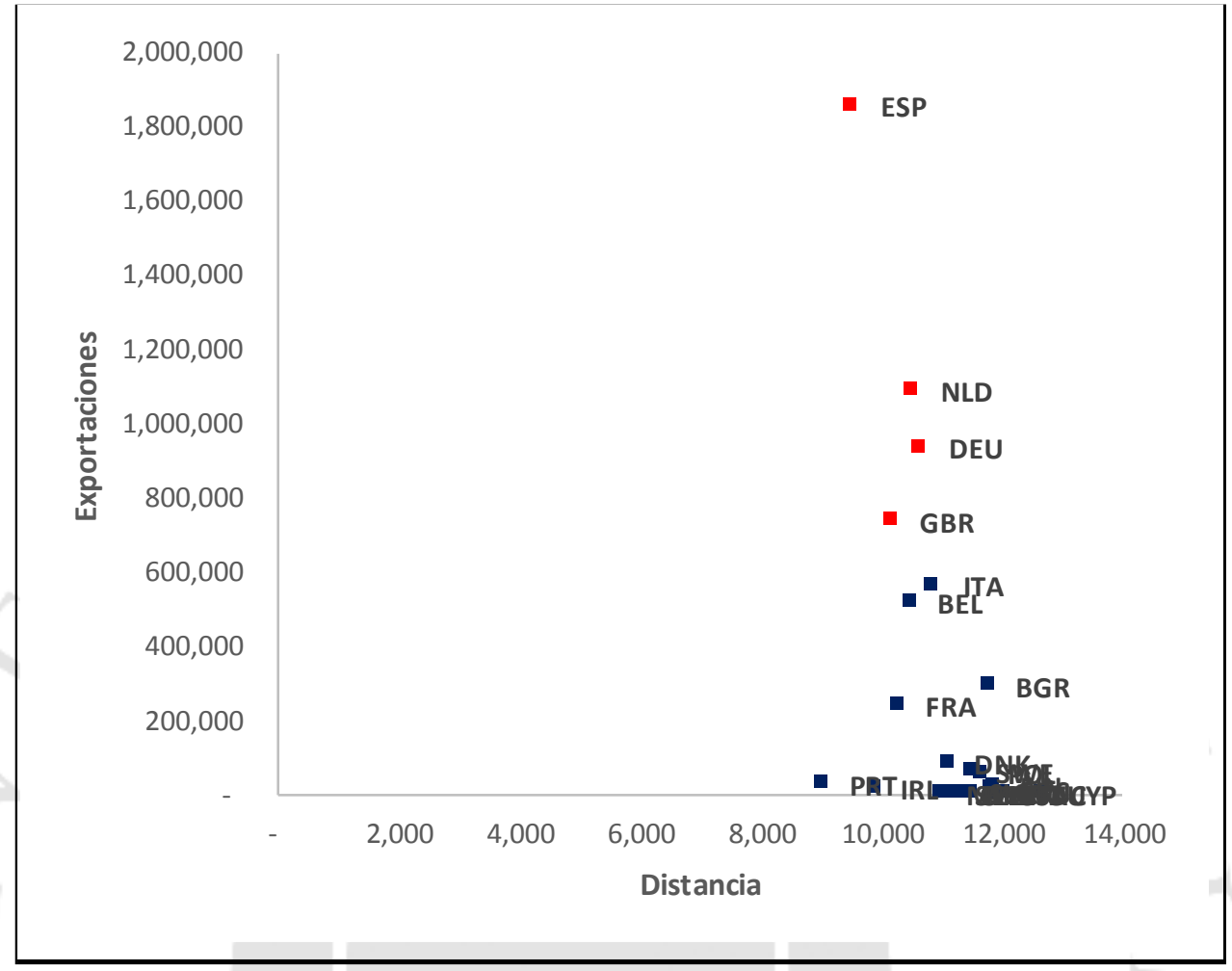

Fuente: Elaboración propia / Adex Data Trade

\subsection{Landlocked}

La variable sin acceso a la costa o Landlocked refleja los sobrecostos que genera no tener acceso al mar. Es decir, las exportaciones son menores a países que presentan esta dificultad. Por ejemplo, Eslovaquia, Austria, Hungría, República Checa y Luxemburgo no tienen acceso a la costa y, tal como indica la teoría, son los países que no son tan representativos para las exportaciones peruanas.

\subsection{Lengua común}

Esta variable explica las facilidades de negociación que brinda hablar una misma lengua entre dos países. Es decir, se espera que el compartir un mismo idioma influye de manera positiva sobre las exportaciones. 


\subsection{Tipo de cambio real bilateral}

El tipo de cambio real bilateral explica el precio relativo de los bienes y servicios de una economía respecto a otra. Se espera que una depreciación del TCRB aumente la demanda externa, es decir, las exportaciones ya que los bienes internos se vuelven más caros (en términos reales) en relación con los bienes extranjeros. Por otro lado, ante una apreciación del TCRB se espera lo contrario.

\subsection{Acuerdo comercial}

El acuerdo comercial entre el Perú y la Unión Europea entró en vigencia en el 2013 y se mantiene hasta la actualidad. Los objetivos generales a favor de las exportaciones peruanas precisan en garantizar el acceso preferencial permanente a la UE, incrementarlas y diversificarlas, propiciar el desarrollo de economía a escala y elevar la productividad de las empresas. Así mismo, en la actualidad, el 95\% de los productos peruanos ingresa a la UE sin pagar arancel y, en el año 2021 lo hará al 100\%. Por lo tanto, se espera un impacto positivo del acuerdo comercial sobre las exportaciones peruanas..

\subsection{Términos multilaterales de resistencia comercial}

Esta variable explica las rémoras comerciales, es decir, dichos elementos que elevan el coste de las transacciones para ambos países. La propuesta dada por Anderson y Wincoop (2003) para considerar los TMRC en la ecuación de gravedad consistía en promediar las barreras arancelarias entre los países que comercian y tratarlas como un elemento que eleva el coste de transporte para países que comercian. Sin embargo, un método alternativo para hallar el TMRC, y que será utilizado en esta investigación, es a través del índice de lejanía del país importador propuesto por Head y Mayer (2003). 


\section{ANÁLISIS Y DISCUSIÓN DE RESULTADOS}

A continuación, se presentará el modelo utilizado, sus dificultades y el análisis de los resultados.

El modelo usado analiza la relación comercial entre el Perú y los veinte y ocho países de la Unión Europea; sin embargo, la variable distancia no presentó relación, respecto al signo esperado, con la teoría de la ecuación de gravedad además que no es estadísticamente significativa (Anexo 3). Según (Urcia Erazo Maria Cecilia, 2016) este problema podría estar vinculado a la presencia de valores atípicos o al tamaño de la muestra de países (p. 49). Ante la dificultad, se incluyeron once países (Anexo 4) al análisis para solucionar la significancia económica y estadística de la variable distancia.

Por otro lado, la crítica hecha por (Head, 2000) indica que el PBI tiende a incrementar el $\mathrm{R}^{2}$ de las regresiones ya que es difícil imaginar un mundo en el que los países grandes no negocien entre sí. Además, existe causalidad dado que las exportaciones e importaciones son parte del PBI (p. 8). Por lo tanto, se reemplazó la variable PBI por PBI per cápita para el Perú y los países de la Unión Europea en busca de mitigar el problema.

Finalmente, la ecuación representativa de las exportaciones peruanas a la UE está definida de la siguiente manera:

$\log X$ ijt $=\log$ PBI it $+\log$ PBI jt + Log Distancia ijt + ACUE $+\log$ TCRB

$$
\text { ijt + Landlocked + Lejanía j }
$$

Donde:

X ijt: Logaritmo de las exportaciones del Perú a los países socios de la UE

PBI it: Logaritmo del PBI per cápita del Perú

PBI jt: Logaritmo del PBI per cápita de los países de la UE

Distancia ijt: Logaritmo de la distancia entre el Perú y el país de la UE

ACUE: Acuerdo Comercial entre el Perú y la UE 
TCRB ijt: Tipo de Cambio Real Bilateral del Perú con respecto al país de la UE

Landlocked: Acceso restringido a la costa

Lejanía j: Logaritmo de los TMRC de los países de la UE

Siguiendo el capítulo 7, se realizaron las siguientes pruebas para obtener la especificación adecuada del modelo.

Primero, se realizaron los diferentes test de raíz unitaria para identificar el problema de estacionariedad. Estas indicaron que las variables en logaritmo no presentaban raíz unitaria.

Luego, para el tratamiento del problema de la multicolinealidad entre las variables explicativas se observó el R cuadrado, este indica el valor de 77 por ciento lo cual indica, a primera vista, la inexistencia de multicolinealidad. Así mismo, se realizó la prueba VIF para observar si existe el problema de multicolinealidad entre las variables explicativas; si tomamos el valor VIF, en conjunto, es menor a 10, por lo tanto no existe multicolinealidad. Además, la tolerancia, 1/VIF, no es cercano a cero (Anexo 5). En resumen podemos inferir que no existe el problema de multicolinealidad.

Posteriormente se realizó la prueba de Hausman para tener conocimiento sobre el tipo de panel data que se debe de utilizar. El resultado indica que con una prob>chi2 de 0.8374 se rechaza la hipótesis nula (Anexo 6), es decir, el modelo a seguir es efectos aleatorios.

Luego se hizo el test de Wald para identificar si existe o no el problema de heterocedasticidad en el modelo. El resultado indica que sí existe ya que se rechaza la hipótesis nula con un prob < chi2 de 0.000 (Anexo 7)

Finalmente se realizó el test de Wooldridge para explicar si el problema de autocorrelación está presente en el modelo. El resultado obtenido indica, con un prob > F de 0.0055 , que se rechaza la hipótesis nula, es decir, sí existe problema de autocorrelación de primer orden (Anexo 8).

Después de haber corregidos el problema de multicolinealidad, heterocedasticidad y autocorrelación de primer orden, los resultados son los siguientes: 
Tabla 7.1

Resultados econométricos

\begin{tabular}{|l|c|}
\hline$X_{\text {ijt }}$ & Efectos Aleatorios \\
\hline PBI it & $0.4630437 * * *$ \\
\hline PBI jt & $1.20837 * * *$ \\
\hline Distancia ijt & $-1.809731 * * *$ \\
\hline ACUE & $0.1397409 * * *$ \\
\hline TCRB ijt & $-0.3853219 * * *$ \\
\hline Landlocked & $-2.429572 * * *$ \\
\hline Lejanía j & -0.0433541 \\
\hline
\end{tabular}

Nota: $* * * \mathrm{p}<0.01, * * \mathrm{p}<0.05 \mathrm{y} * \mathrm{p}<0.1$. Mayor detalle en el Anexo 9

En suma los estimadores de las variables están alineados con la teoría económica; además, éstas son significativas de manera individual al nivel del 1 por ciento salvo la variable lejanía del país importador, lo cual indica que no influyó sobre las exportaciones peruanas a la UE en el periodo de estudio.

Tal como indica la teoría, se espera que se comercie más con los países grandes que con los pequeños. Por lo tanto, el PBI per cápita del socio comercial es determinante para las exportaciones peruanas, su coeficiente indica que ante un aumento de 10 por ciento del ingreso per cápita del país importador genere un incremento del 12 por ciento de la variable dependiente. Así mismo, el PBI per cápita del Perú es significativo de modo que el aumento en 10 por ciento ocasiona un crecimiento de 4 por ciento de las exportaciones peruanas manteniendo lo demás constante. Estos resultados indican que la renta per cápita del importador es más fuerte que la del Perú debido a que la mayor parte de las exportaciones peruanas tienen como destino países desarrollados. Este resultado es similar al encontrado por los autores Bacaria-Colom et al. (2013) y Correia (2008) cuyas investigaciones se basan en el acuerdo comercial entre países como México y Colombia en relación con la Unión Europea respectivamente, observándose que la elasticidad del estimador del ingreso del país exportador e importador es positiva y está en torno a la unidad. 
De igual manera, siguiendo la teoría del modelo de gravedad, la distancia, como proxy de los costos de transporte, es determinante para las exportaciones peruanas, su coeficiente explaya que un incremento en 10 por ciento de la distancia entre dos países implica un decrecimiento del 18 por ciento, ceteris paribus. Esto es resultado de la ineficiencia logística, para ser más claros, la inexistencia de parques industriales eficientes que ayuden a mejorar los costos de transporte. Los valores de este coeficiente es compartido con la mayoría de los autores de los cuales se basa la presente investigación.

Respecto al acuerdo comercial entre el Perú y la UE, ésta indica que sí ha sido significativa para las exportaciones peruanas y está alineada a la teoría económica ya que influye de manera positiva. Según los resultados encontrados, si el Perú pone en vigencia un acuerdo comercial con un determinado país, sus exportaciones incrementarán en 13 por ciento, ceteris paribus. Del mismo modo, el coeficiente encontrado evidencia una clara similitud con los autores Correia (2008), Bacaria-Colom (2013), Ordoñez et al. (2015) y Serrano, Martínez y Rodríguez (2015) que evaluaron el impacto de un acuerdo comercial y encontraron un impacto positivo sobre el flujo comercial.

Examinando la variable landlocked o sin acceso a la costa, el resultado comprobó que esta influyó de manera negativa sobre las exportaciones peruanas y siguió la teoría económica puesto que si uno de los países no tiene acceso a la costa los costes de transporte se elevan. Su coeficiente indica que si el Perú exporta a un país sin acceso al mar, sus exportaciones disminuirían en un 24 por ciento. Tal es el caso de aquellas economías como Luxemburgo y Austria que, a pesar de tener un ingreso per cápita mayor al promedio de países estudiados, no representan a los principales destinos de las exportaciones del Perú.

Para finalizar, el resultado del tipo de cambio real bilateral tiene relación significativa y negativa tal como indica la teoría. Su coeficiente evidenció que ante una apreciación del tipo de cambio real bilateral en 1 por ciento, las exportaciones peruanas disminuyan en 0.3 por ciento, manteniendo lo demás constante. Es decir, ante una disminución del tipo de cambio real se espera que las exportaciones peruanas actúen de la misma manera porque a partir del año 2011 hasta el 2017 ocurrió un descenso del tipo de cambio nominal, teniendo como causa principal a la crisis del euro. 


\section{RECOMENDACIONES}

A lo largo del siglo XXI el Perú ha aumentado sus exportaciones, en parte, a su apertura comercial con el mundo a través de acuerdos comerciales, tratados de libre comercio, acuerdos de complementación económica y acuerdos de integración comercial, en especial, con la Unión Europea. Sin embargo, como hemos observado a lo largo del caso de estudio, es necesario el enfoque de las políticas económicas hacia otros aspectos que también son significativas e impactan en las exportaciones peruanas debido a que el efecto de los tratados comerciales no es de forma inmediata y existen externalidades, como crisis mundiales o variación de los precios internacionales, que no la permiten actuar de forma eficaz.

En tal sentido, las recomendaciones estarían enfocadas, en primer lugar, al fortalecimiento de las relaciones comerciales con la UE ya que este bloque económico incorpora países con un elevado PBI per cápita como Luxemburgo, Dinamarca, Suecia e Irlanda pero que no son los principales destinos de las exportaciones del Perú, por lo tanto, podrían ser considerados como oportunidades no aprovechadas por los productos peruanos. Así mismo, gestionar un plan de diversificación productiva para no depender de los minerales ni del consumo periódico de estos. Por lo tanto, las exportaciones peruanas dejarían de depender de los productos mineros tradicionales y se encaminarían por la mayor exportación de productos no tradicionales y aprovechar las oportunidades que brinda el mercado de la UE.

Finalmente, ante el elevado impacto de la variable distancia, la inversión en infraestructura y el desarrollo de parques industriales es determinante en busca de atenuar el costo de transporte de las mercancías para que estas sean competitivas en el mercado exterior ya que, siguiendo a (COMEXPERU, 2018), los parques industriales son espacios habilitados para la actividad industrial y son determinantes para promover el crecimiento económico debido a que generan incentivos para la investigación (párr. 1). 


\section{REFERENCIAS}

Bacaria-Colom, J., Osorio-Caballero, M. I., y Artal-Tur, A. (2013). Evaluación del Acuerdo de Libre Comercio México-Unión Europea mediante un modelo gravitacional. Economia Mexicana, Nueva Epoca, 22(3), 143-163. Recuperado de http://www.economiamexicana.cide.edu/num_anteriores/Cierre-

1/04_EM_Jordi_Bacaria_(143-163).pdf

COMEXPERU. (2018.). ¿Parques industriales? Recuperado de https://semanariocomexperu.wordpress.com/parques-industriales/

Correia, J. (2008). The Determinants of Colombian Exports: An Empirical Analysis Using the Gravity Model. Desarrollo y Sociedad, 165-205. https://doi.org/https://doi.org/10.13043/dys.61.5

Deardorff, A. V. (1995). Determinants of Bilateral Trade: Does Gravity Work in a Neoclassical World? Recuperado de https://www.nber.org/chapters/c7818.pdf

Földvár, P. (2006). The Economic Impact of the European Integration on the Netherlands A Quantitative Analysis of Foreign Trade and Foreign Direct Investments, 1-143. Recuperado de https://s3.amazonaws.com/academia.edu.documents/37467491/thesisFoldvari.pdf? AWSAccessKeyId=AKIAIWOWYYGZ2Y53UL3A\&Expires=1542611482\&Sign ature $=X M b 0 w e j 8 a C K G A e S 2 x 44$ syURWzAM\%3D\&response-contentdisposition=inline\%3B filename\%3DThe_economic_impact_of_the_Euro

Head, K. (2000). Gravity for Beginners *. Recuperado de https://artnet.unescap.org/tid/artnet/mtg/gravity10_reading1.pdf

Krugman, P. R., Obstfeld, M., y Melitz, M. J. (2012). Economía internacional Teoría y política. Recuperado de file:///Users/israellaos/Downloads/Economia Internacional. 9Ed. Krugman, 2012.pdf

Mayorga, M. M., y Muñoz, E. S. (2000). LA TÉCNICA DE DATOS DE PANEL UNA GUÍA PARA SU USO E INTERPRETACIÓN. Recuperado de https://s3.amazonaws.com/academia.edu.documents/8300249/mayorga y munoz tecnica de datos de 
panel.pdf?AWSAccessKeyId=AKIAIWOWYYGZ2Y53UL3A\&Expires=1542614 730\&Signature=zyrfcPTx1J\%2FrJ46KHBIdjBYVROY\%3D\&response-contentdisposition=inline $\% 3 \mathrm{~B}$

MINCETUR. (2018a). Acuerdo Comercial entre Perú y la Unión Europea. Recuperado de

http://www.acuerdoscomerciales.gob.pe/index.php?option=com_content\&view=ca tegory\&layout=blog\&id=50\&Itemid=73

MINCETUR. (2018b). COMERCIO BILATERAL PERÚ - UNIÓN EUROPEA, 1-4. Recuperado de file:///Users/israellaos/Downloads/RBC Perú UE(28) (1).pdf

Sierra Fernández, M. del P., y Martínez Campillo, A. (2009). Impacto del proceso de integración europea sobre las exportaciones de Castilla y León (1993-2007): un análisis econométrico a partir de la ecuación de gravedad. Estudios de Economía Aplicada, 27(3), $=1-34$ Recuperado de https://dialnet.unirioja.es/descarga/articulo/3227554.pdf

Urcia Erazo Maria Cecilia. (2016). Aplicacion del modelo de gravedad para el analisis de los determinantes del flujo de importaciones peruanas de origen asiatico en el periodo 2000-2014. Pontificia Universidad Católica Del Perú, 151. Recuperado de http://tesis.pucp.edu.pe/repositorio/handle/123456789/8484 


\section{BIBLIOGRAFÍA}

Anderson, J. E. (1979). A Theoretical Foundation for the Gravity Equation. The American Economic Review, 69, 106-116. https://doi.org/10.2307/1802501

Anderson, J. E., y Wincoop, E. van. (2003). Gravity with \{Gravitas $\}$ : $\{$ A $\}$ \{Solution $\}$ to the $\{$ Border $\}$ \{Puzzle\}. American Economic Review, 93(1), 170-192. https://doi.org/10.1257/000282803321455214

Ball, R. J., y Linnemann, H. (1967). An Econometric Study of International Trade Flows. The Economic Journal, 77(306), 366. https://doi.org/10.2307/2229319

Bergstrand, J. H. (1985). The Gravity Equation in International Trade: Some Microeconomic Foundations and Empirical Evidence. The Review of Economics and Statistics, 67(3), 474-481. https://doi.org/10.2307/1925976

Bergstrand, J. H. (1989). The Generalized Gravity Equation, Monopolistic Competition, and the Factor-Proportions Theory in International Trade. The Review of Economics and Statistics, 71(1), 143-153. https://doi.org/10.2307/1928061

Cafiero, J. A. (2005). Modelos Gravitacionales para el Análisis del Comercio Exterior. Recuperado de http://cei.mrecic.gob.ar/userfiles/4 modelos gravitacionales para el análisis del comercio.pdf

Correia, J. (2008). The Determinants of Colombian Exports: An Empirical Analysis Using the Gravity Model. Desarrollo y Sociedad, 165-205. https://doi.org/https://doi.org/10.13043/dys.61.5

Deardorff, A. V. (1998). Determinants of Bilateral Trade: Does Gravity Work in a Neoclassical World?, 267-293. https://doi.org/10.1142/9789814340373_0024

Eaton, J., y Kortum, S. (2002). Technology, Geography, and Trade. Econometrica, 70(5), 1741-1779. https://doi.org/10.1111/1468-0262.00352 
Evenett, S. J., y Keller, W. (2002). On Theories Explaining the Success of the Gravity Equation. Journal of Political Economy, 110(2), 281-316. https://doi.org/10.1086/338746

Head, K., y Mayer, T. (2013). Gravity Equations: Workhorse,Toolkit, and Cookbook, 70. Recuperado de http://www.cepii.fr/pdf_pub/wp/2013/wp2013-27.pdf

Helpman, E., y Krugman, P. R. (1985). Market structure and foreign trade : increasing returns, imperfect competition, and the international economy. MIT Press. Recuperado de https://books.google.com.pe/books?hl=es\&lr=\&id=KB_bO8w4JgoC\&oi=fnd\&pg $=$ PR11\&dq=HELPMAN,+E.+y+KRUGMAN,+P.+(1985):+Market+Structure+and +Foreign+Trade:+Increasing+Returns,+Imperfect+Competition+and+the+Internati onal+Economy.+Cambridge:+MIT+Press\&ots=dFsruk-

VKS\&sig=JjykWXfPzepo9bvXcybyUcm8fxc\#v=onepage\&q=HELPMAN\%2C E. y KRUGMAN\%2C P. (1985) \%3A Market Structure and Foreign Trade\%3A Increasing Returns\%2C Imperfect Competition and the International Economy. Cambridge\%3A MIT Press\&f=false

Krugman, P., Cooper, R. N., y Srinivasan, T. N. (1995). Growing World Trade: Causes and Consequences. Brookings Papers on Economic Activity, 1995(1), 327-377. https://doi.org/10.2307/2534577

McCallum, J. (1995). National \{Borders $\}\{$ Matter $\}:\{$ Canada $\}-\{U\} .\{S\} .\{$ Regional $\}$ \{Trade\} \{Patterns\}. American Economic Review, 85(3), 615-623. https://doi.org/10.2307/2118191

Ordóñez Tórrez, B., Magdiel, K., Rojas Roque, B., Adrián, C., Rojas Salinas, B., Walkiria Tutor, Y., y Yáder Alberto Avilés Peralta, L. (2015). Factores que explican los flujos de exportaciones de Nicaragua hacia sus quince principales destinos comerciales: un análisis basado en el modelo gravitacional del periodo 1009-2013. Recuperado de http://riul.unanleon.edu.ni:8080/jspui/bitstream/123456789/4015/1/229004.pdf 
Pöyhönen, P. (1963). A Tentative Model for the Volume of Trade between Countries. Weltwirtschaftliches Archiv, 93-100. https://doi.org/10.2307/40436776

Serrano, C., Martínez, A., Rodríguez, A., \& Salazar, S. (2015). Evaluación de los efectos del Tratado de Libre Comercio entre la Unión Europea y México en el comercio bilateral y la inversión. Recuperado de https://www.bbvaresearch.com/wpcontent/uploads/2015/05/DT15-13_TLCUEM.pdf

Tinbergen, J. (1962). Shaping the World Economy: Suggestions for an International Economic Policy. New York, United States of America: The Twentieth Century Fund. Recuperado de hdl.handle.net/1765/16826. 


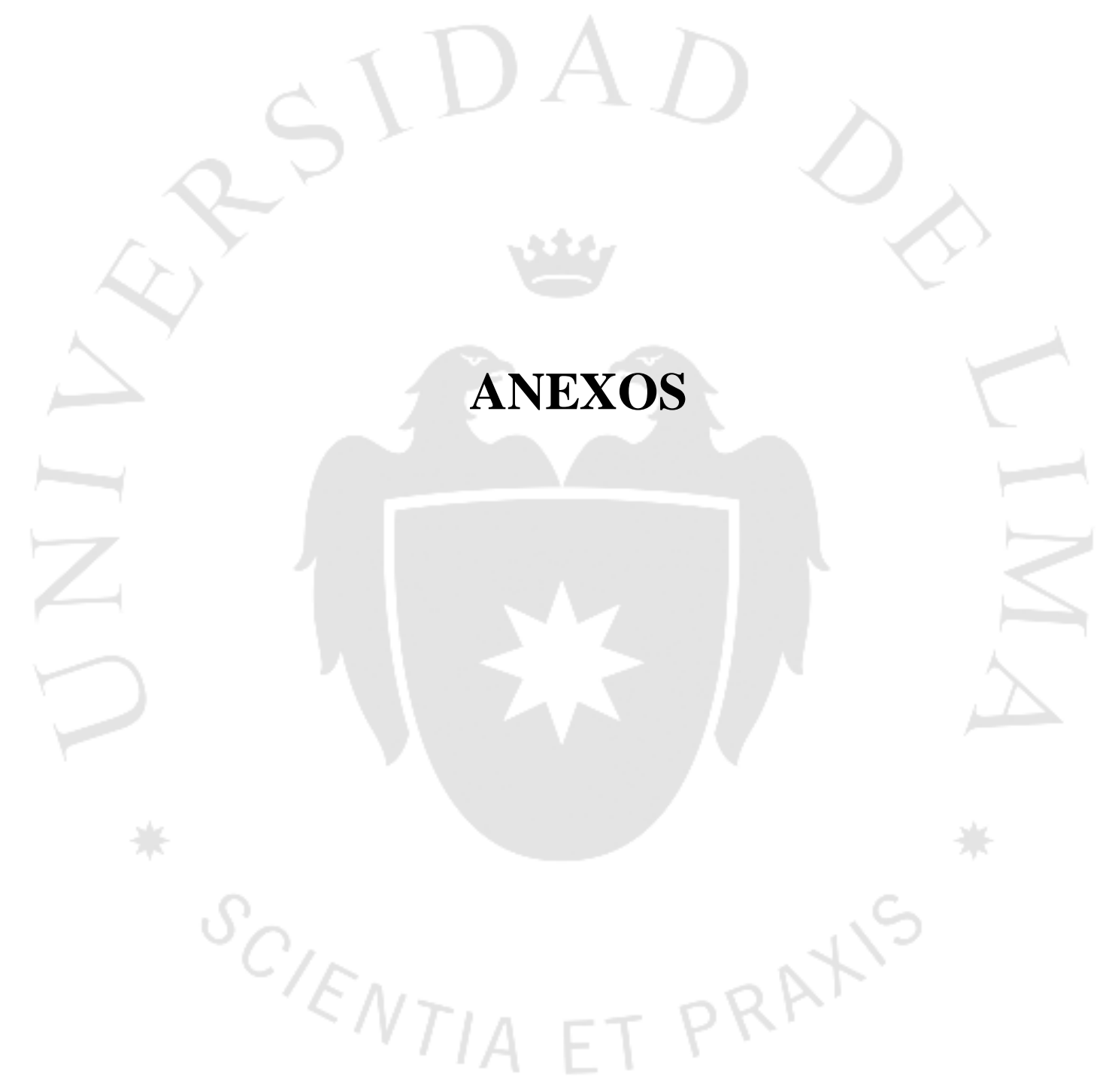




\section{Anexo 1: Tipo de cambio real bilateral}

Siguiendo la guía metodológica de la Nota Semanal del Banco Central de Reserva del Perú el tipo de cambio real bilateral se calcula de la siguiente manera:

$$
\text { TCRbil }=\frac{\text { E } / .}{I P \text { U.i } . \times I P C_{i}}
$$

Donde:

S/.

E U.M. : Índice de Tipo de cambio nominal del nuevo sol respecto a la unidad monetaria del país i

$I P C$ : Índice de precios al consumidor del país i

IPCPerú : Índice de precios al consumidor del Perú

\section{Anexo 2: Índice de lejanía}

El índice de lejanía es el proxy a la variable TRMC. Tomando a Head y Mayer (2003) como referencia, este se mide de la siguiente manera:

$$
\text { Lejanía } \mathrm{i}=\Sigma \underline{\text { distancia } \mathrm{ij}}
$$

Donde:

Distancia ij: Distancia entre el país i y j

PBI j: PBI del país j

PBI mundo: PBI del mundo 


\section{Anexo 3: Primera corrida econométrica}

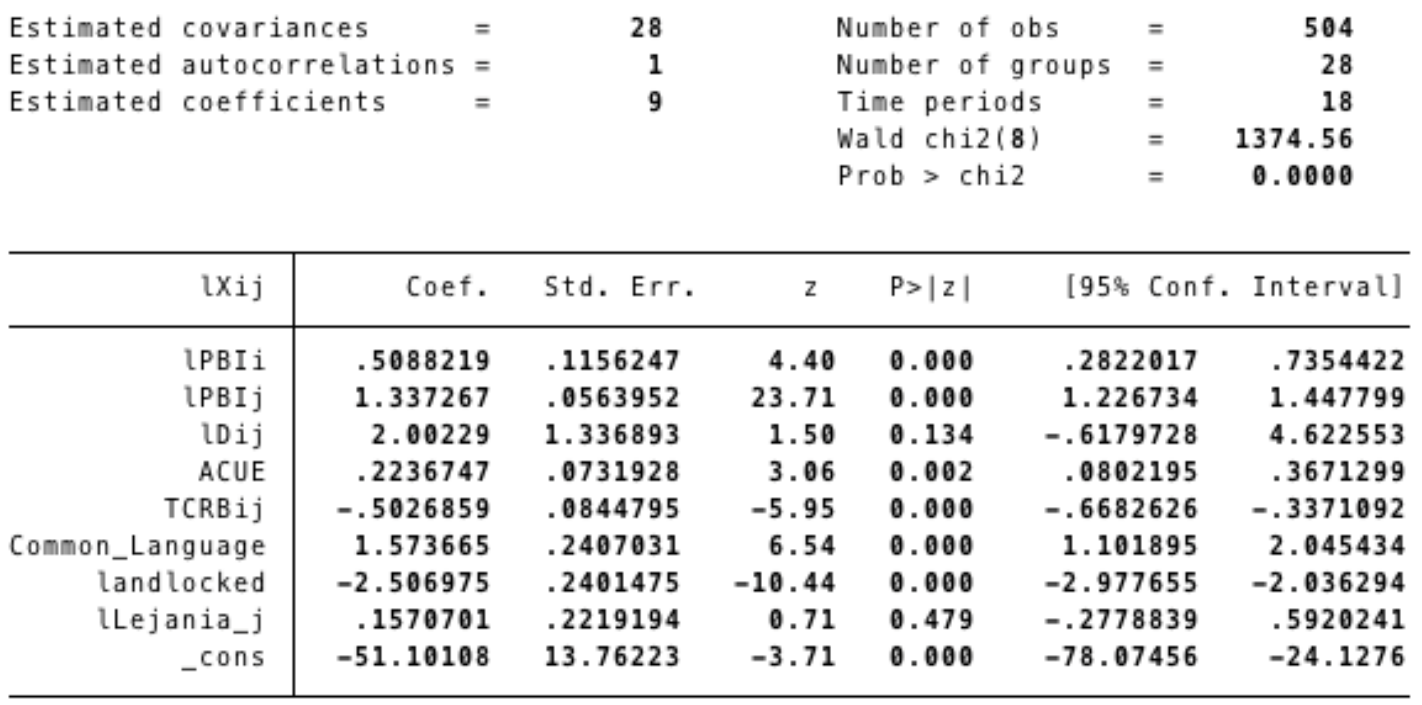

\section{Anexo 4: Países incluidos}

\begin{tabular}{|c|}
\hline Países adheridos al análisis \\
\hline Brasil \\
\hline Canadá \\
\hline Suiza \\
\hline Chile \\
\hline China \\
\hline Ecuador \\
\hline India \\
\hline Japón \\
\hline Corea del Sur \\
\hline Panamá \\
\hline Estados Unidos \\
\hline Pún \\
\hline
\end{tabular}




\section{Anexo 5: Prueba de multicolinealidad}

\begin{tabular}{|c|c|c|c|c|c|c|}
\hline Source & SS & $d f$ & MS & Number of obs & $=$ & 702 \\
\hline Model & 6154.36484 & 7 & 879.194978 & Prob > F & $=$ & 0.0000 \\
\hline Residual & 1801.67445 & 694 & 2.5960727 & R-squared & $=$ & 0.7735 \\
\hline Total & 7956.0393 & 701 & 11.3495568 & Root MSE & $=$ & 1.6112 \\
\hline
\end{tabular}

\begin{tabular}{r|rrrrrr}
\hline LXij & Coef. & Std. Err. & $t$ & P $>|t|$ & [95\% Conf. Interval] \\
\hline LPBIi & .2616509 & .1533589 & 1.71 & 0.088 & -.0394522 & .5627539 \\
LPBIj & 1.345677 & .0351634 & 38.27 & 0.000 & 1.276638 & 1.414717 \\
LDij & -2.075655 & .1175011 & -17.66 & 0.000 & -2.306355 & -1.844954 \\
ACUE & .1399258 & .158547 & 0.88 & 0.378 & -.1713635 & .4512151 \\
TCRBij & -.4866652 & .0498336 & -9.77 & 0.000 & -.5845078 & -.3888226 \\
Landlocked & -1.936814 & .1727455 & -11.21 & 0.000 & -2.275981 & -1.597648 \\
LLejania_j & .6944853 & .5198497 & 1.34 & 0.182 & -.3261813 & 1.715152 \\
_Cons & -31.56588 & 9.87954 & -3.20 & 0.001 & -50.96325 & -12.16851 \\
\hline
\end{tabular}

. estat vif

\begin{tabular}{r|rr} 
Variable & VIF & $1 /$ VIF \\
\hline LPBIi & 1.70 & 0.588125 \\
ACUE & 1.62 & 0.619039 \\
ULejania_j & 1.52 & 0.657331 \\
TCRBij & 1.33 & 0.754619 \\
LPBIj & 1.15 & 0.868477 \\
LDij & 1.06 & 0.946712 \\
landlocked & 1.05 & 0.951986 \\
\hline Mean VIF & 1.35 &
\end{tabular}

\section{Anexo 6: Prueba de Hausman}

\begin{tabular}{|c|c|c|c|c|}
\hline & \multicolumn{2}{|c|}{$\longrightarrow$ Coefficients -} & \multirow{3}{*}{$\begin{array}{c}(b-B) \\
\text { Difference }\end{array}$} & \multirow{3}{*}{$\begin{array}{c}\left.\operatorname{sqrt}\left(\operatorname{diag}_{\left(V_{-} b-V_{-} B\right.}\right)\right) \\
\text { S.E. }\end{array}$} \\
\hline & (b) & (B) & & \\
\hline & $\mathrm{FE}$ & RE & & \\
\hline LPBI i & .5192404 & .379957 & .1392834 & .1025656 \\
\hline lPBI j & 1.017498 & 1.220983 & -.203485 & .154291 \\
\hline ACUE & .2221597 & .2115403 & .0106195 & .0176153 \\
\hline TCRBij & -.4777188 & -.5423837 & .0646649 & .0690173 \\
\hline ULejania_j & .5556438 & .5828879 & -.0272441 & .0391889 \\
\hline
\end{tabular}

$b=$ consistent under $\mathrm{Ho}_{0}$ and $\mathrm{Ha}$; obtained from xtreg $B=$ inconsistent under $\mathrm{Ha}$, efficient under $\mathrm{Ho}$; obtained from xtreg

Test: Ho: difference in coefficients not systematic

$$
\begin{array}{rlrl}
\operatorname{chi2}(5) & = & (b-B) \cdot\left[\left(V_{-} b-V_{-} B\right) \wedge(-1)\right](b-B) \\
& = & & 2.08 \\
\text { Prob }>\text { chi2 } & = & & 0.8374
\end{array}
$$




\section{Anexo 7: Prueba de heterocedasticidad}

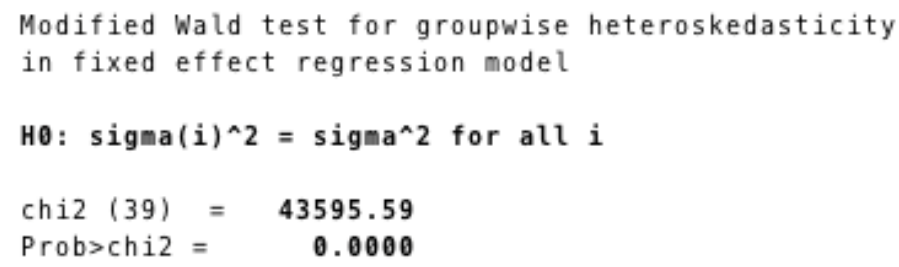

\section{Anexo 8: Prueba de autocorrelación de primer orden}

Wooldridge test for autocorrelation in panel data HO: no first order autocorrelation
Fi 1,
38) $=$
8.668
Prob $>F=$
0.0055

Cross-sectional time-series FGLS regression

Coefficients: generalized least squares

Panels: heteroskedastic

Correlation: common AR(1) coefficient for all panels $(0.8001)$

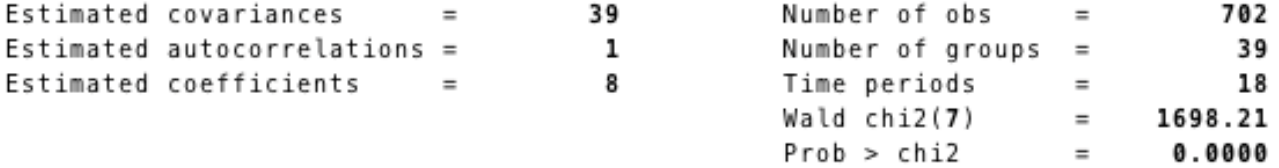

\begin{tabular}{r|rrrrrr}
\hline LXij & Coef. & Std. Err. & $z$ & P $>|z|$ & [95\% Conf. Interval] \\
\hline LPBIi & .4630437 & .081868 & 5.66 & 0.000 & .3025854 & .623502 \\
LPBIj & 1.208371 & .0416327 & 29.02 & 0.000 & 1.126772 & 1.28997 \\
LDij & -1.809731 & .0803163 & -22.53 & 0.000 & -1.967148 & -1.652314 \\
ACUE & .1397409 & .0397094 & 3.52 & 0.000 & .061912 & .2175699 \\
TCRBij & -.3853219 & .032714 & -11.78 & 0.000 & -.4494402 & -.3212035 \\
Landlocked & -2.429572 & .2932871 & -8.28 & 0.000 & -3.004405 & -1.85474 \\
LLejania_j & -.0433541 & .1885279 & -0.23 & 0.818 & -.412862 & .3261538 \\
_Cons & -20.99407 & 4.027238 & -5.21 & 0.000 & -28.88731 & -13.10083 \\
\hline
\end{tabular}

